Arthroskopie 2009 $\cdot 22: 173-174$

DOI 10.1007/s00142-009-0534-y

Online publiziert: 05. Juli 2009

(c) Springer Medizin Verlag 2009

A. Imhoff

Abt. und Poliklinik für Sportorthopädie, Klinikum rechts der Isar, TU München

\title{
Die AGA in der neuen DGOU?
}

letzten Jahr nun zu Doppelmitgliedern ihrer Fachgesellschaft und der DGOU geworden sind.

Mehrere Sektionen und Arbeitsgemeinschaften der DGOOC und DGU haben sich in den letzten Monaten bereits zusammengeschlossen und den Antrag auf Aufnahme in die DGOU gestellt. Dazu gehört die Sektion für Grundlagenforschung der DGOOC oder die Vereinigte Sektion für Physikalische Therapie und Rehabilitation. Andere Anträge liegen nun auch von den Sektionen Fußchirurgie und Sportorthopädie vor.

Um in der neuen DGOU bereits von Beginn an mitgestalten und mitbestimmen zu können, muss die AGA sich zur DGOU hin bewegen. Unterschiedliche Kulturen und heterogene Bedürfnisse existierender Vereine, Arbeitsgruppen und Sektionen in den Fachgesellschaften werden ein Zusammengehen und eine Zusammenarbeit nicht einfach machen, aber wir müssen jetzt die Chance dazu ergreifen.

Der Vorstand der AGA glaubt, dass wir als weltweit größte Arthroskopiegesellschaft mit über 2500 Arthroskopeuren aus den deutschsprachigen Ländern auch die Thematik der Arthroskopie in der neuen Dachgesellschaft vertreten müssen. Wir möchten das rasch expandierende Spezialwissen auf dem Gebiet der Arthroskopie in diese Gesellschaft und in die Kongresse einbringen. Wir denken dabei an die Organisation gemeinsamer Kurse und insbesondere die Strukturierung des Angebots zur Fort- und Weiterbildung auf dem Gebiet der Arthroskopie, an die Organisation von Spezialsitzungen auf den Jahrestagungen unter Einbeziehung der Kom- petenz unseres Bundesverbandes Ambulanter Arthroskopeure (BVASK).

In den letzten Wochen haben viele Gespräche mit den Vertretern des neuen Vorstands der DGOU stattgefunden und die Wege sind für die AGA offen; es ist der ausdrückliche Wunsch der DGOU, mit uns zusammen zu arbeiten, unsere Kompetenz in diese Dachgesellschaft einzubringen und die Arthroskopie nicht als Technik alleine, sondern die arthroskopischen chirurgischen Verfahren topographiebezogen in die Aus- und Weiterbildung des Fachs kompetent zu integrieren.

Die DGOU bietet nun ein Modell für Sektionen wie die AGA an (Typ II): Eingetragener Verein mit „Status Sektion der DGOU“. Die Ziele der Vereinssatzung der AGA müssen mit denen der DGOU-Satzung übereinstimmen und die besondere Kooperation mit den Organen der DGOU beinhalten. Der Verein bzw. die AGA wird außerordentliches Mitglied der DGOU sein und hat als außerordentliches Mitglied aktives Wahlrecht und einen Vertreter im Fachbeirat im Gesamtvorstand. Ist der Vertreter auch gleichzeitig ordentliches Mitglied in der DGOU, hat er auch Stimmrecht und ist wählbar. Damit können die AGA-Mitglieder in Ausschüssen, Kommissionen und Arbeitsgemeinschaften der DGOU Mitglied werden. Wir haben die Möglichkeit der Mitarbeit bei der Gestaltung von Kongressen und erhalten Unterstützung bei Fort- und Weiterbildungsveranstaltungen und Stipendienvergabe wie auch bei den Publikationen der DGOU.

Ich finde es richtig, dass der Vorstand der AGA den Antrag zur Aufnahme als 
Sektion der DGOU (Sektion Typ II) am 06.06.2009 gestellt hat, damit im Laufe des Sommers die Beitrittsverhandlungen starten und die Mitglieder anlässlich der Mitgliederversammlung in Leipzig über das weitere Vorgehen informiert werden können. Nur durch aktive Mitarbeit beim Bau des neuen Hauses DGOU kann die AGA ihren Stellenwert im zusammenwachsenden Fach Orthopädie und Unfallchirurgie ausbauen.

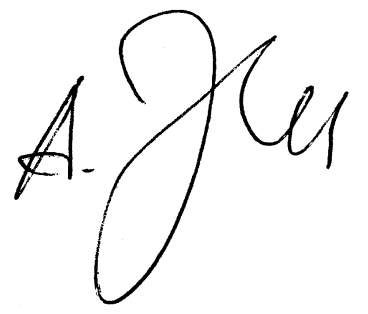

Prof. Dr. A.B. Imhoff

Schriftleiter Zeitschrift Arthroskopie AGA-Sekretär

\section{Korrespondenzadresse \\ Prof. Dr. A.B. Imhoff}

Abt. und Poliklinik für Sportorthopädie, Klinikum rechts der Isar, TU München Connollystr. 32, 80809 München sportortho@lrz.tum.de

Interessenkonflikt. Der korrespondierende Autor gibt an, dass kein Interessenkonflikt besteht.

\section{Petersen, Wolf; Zantop, Thore Das vordere Kreuzband} Grundlagen und aktuelle Praxis der operativen Therapie

Köln: Deutscher Ärzte-Verlag 2009, 250 S., (ISBN 978-3-7691-0562-9), 149.00 EUR

Diese Monographie füllt eine Lücke in den Büchern zum Kniegelenk, weil das vordere Kreuzband gemessen an der Zahl der Publikationen im Mittelpunkt der Forschung am Kniegelenk steht. Wenn auch in den letzten Jahre viele kontrovers diskutierten Fragen im Hinblick auf das vordere Kreuzband gelöst sind, hat sich sowohl im Bereich der Diagnostik mit dreidimensionalen Darstellungen im CT und neuen MRT-Ebenen und MRTSequenzen eine differenzierte präoperative Diagnostik ergeben. Eine Rückbesinnung auf die Anatomie erlaubt eine bessere Operationstechnik, da fehlpositionierende Bohrkanäle einer der häufigsten Gründe für Revisionsoperationen nach VKB-Rekonstruktionen darstellen.

Prof. Wolf Petersen und Dr. Thore Zantop haben diese schwierige Aufgabe hervorragend gelöst, in einem konsistenten leicht lesbaren monographischen Werk nicht nur einen aktuellen Literaturüberblick über die verschiedenen Aspekte des vorderen Kreuzbandes darzustellen, sondern auch viele eigene Erfahrungen anatomischer und biomechanischer Untersuchungen und neue Operationstechniken vorzustellen.

So nehmen die anatomischen und biomechanischen Grundlagen des Kniegelenks und speziell des vorderen Kreuzbandes in den einleitenden Kapiteln einen breiten Raum ein. Das nicht nur einfach Bekanntes neu geschrieben wurde, zeigt zum Beispiel auch die ausführliche Darstellung intraartikulärer Rupturmuster und Partialrupturen des VKBs mit der eigenen Klassifikation eines alphanumerischen Codes, wo nun auch die LambdaHeilung des VKBs exakt klassifiziert werden kann. Dabei kommt es bekanntlich nach femoraler Ruptur des AM-Bündels zu einer Auflagerung des Rupturendes auf das HKB, fallweise mit intaktem PL-Bündel, klassifiziert dann als 4e bzw. mit elongiertem teilrupturierten posterolateralen Bündel, klassifiziert als $4 d$, die dann auch folgerichtig bei einer subjektiven Instabilität eine VKB-Rekonstruktion gerechtfertigt ist.
Auch die Unterscheidung der Rupturformen als komplette Ruptur AM-Ruptur, PL-Ruptur mit der Zuordnung Lachman-Test vordere Schublade und Pivotshift ist in einer schönen Tabelle sauber dargestellt und sehr hilfreich. Dass in der Spalte „Floppy VKB“ die an sich richtige HKB-Ruptur-Diagnose erst unten erscheint, könnte beim Überlesen in die falsche Richtung führen.

Das verbesserte Verständnis von Partialrupturen hat nun auch viele Augmentationsmöglichkeiten wieder in Erinnerung gerufen, wurde aber durch die Double bundle-Technik mit der isolierten Rekonstruktion eines der beiden Bündel in den letzten Jahren ins Blickfeld gerückt, weshalb auch hier ein ganzes Kapitel der reinen Augmentation aus der Pittsburgher Autorenschaft gewidmet ist. Interessant sind die Darstellungen der verschiedenen Operationsverfahren, die nebeneinander in einzelnen Kapiteln dargestellt und auch mit kritischen Bemerkungen gewichtet werden. Schließlich freut es den Rezensent auch, dass die immer häufiger werdenden Begleitverletzungen wie Knorpelschäden, Meniskusläsionen und beginnenden Arthrosen in Kombination mit Läsionen des vorderen Kreuzbandes behandelt werden und auch die immer wichtiger werdende hohe tibiale Korrekturosteotomie als Kombinationstherapie in einzelnen Kapiteln sehr elegant und leicht zu lesen dargestellt wird. In diese Richtung gehen auch die Kapitel zu Komplikationen wie Tunnelweitung, Arthrofibrose nach VKB-Ersatz, Infektionen sowie auch die Revisionsoperationen, die jeweils in ausführlichen Kapiteln umfassend dargestellt und mit hervorragendem Bildmaterial illustriert werden. Etwas schade, aber nicht dem ursprünglichen Ziel des Buches entsprechend, ist die nur noch kurze Abhandlung zur Rehabilitation nach VKB-Rekonstruktionen. Sie ist so kurz, dass man sich auch fragen könnte, ob man sie dem hauptsächlichen Ziel des Buches „Die Grundlage und aktuelle Praxis der operativen Therapie“ darzustellen nicht lieber weglassen würde. Das Buch ist sehr angenehm zu lesen und entführt den Leser auf über 250 Seiten in ein sehr spezielles, aber immer wichtigeres Gebiet, dass nicht nur für den Spezialisten wichtig ist, sondern auch jedem Facharzt und jedem interessierten Assistenzarzt sehr zu empfehlen ist.

Andreas Imhoff (München) 